\title{
When it isn't always lyme: expanding the differential diagnosis for acute-onset polyarthralgia in the West Virginia eastern panhandle
}

Natalie A. Moffett ${ }^{1}$, Rosemarie Lorenzetti ${ }^{1}$

\section{Author Affiliations:}

1. West Virginia University, Morgantown, West Virginia

The authors have no financial disclosures to declare and no conflicts of interest to report.

\section{Corresponding Author}

Natalie A. Moffett

West Virginia University

Morgantown, West Virginia

Email: namoffett@mix.wvu.edu 


\section{Abstract}

This case presentation discusses a 36 year-old female animal care worker presenting with an acute-onset polyarthropathy during the summer months in a Lyme endemic region. Though she appeared to be a good candidate for the diagnosis of Lyme borreliosis, her screening serology reported negative results and alternative diagnoses were considered. Her subsequent diagnosis with parvovirus B19 acts to remind the general practitioner to have confidence in the accuracy of a negative Lyme screen and, upon negative result, to expand the differential to include less common infections including parvovirus B19. It also highlights the need to remember parvovirus B19 in a similar patient who has the potential to be or to become pregnant during the course of her illness.

\section{Key Words}

Lyme disease, Parvovirus B19, Polyarthropathy, Lyme borreliosis screening test, TORCH infections, Appalachia

\section{Introduction}

In an Ixodes tick endemic region like the eastern panhandle of West Virginia, it is not uncommon for health care practitioners to include Lyme disease on the differential for acute-onset polyarthralgias. This is a case of a 36 year-old female presenting with a history of polyarthralgia initially thought to be Lyme disease. A review of this case makes an argument for the inclusion of additional testing and consideration of alternative diagnoses before assuming a diagnosis of Lyme disease.

\section{Case Report}

A 36 year-old female presented to the ED with acute left-sided arm and hand pain that was initially waxing and waning but had increased in severity for the past six hours. Her recent history included a two-week period of intermittent fatigue, myalgias, occasional joint aching and swelling all following a single episode of fever and headache. Her past medical history was significant for a history of multiple zoonotic infections from her work in the animal care industry. She had no history of sexually transmitted infections and had two school-age children and one 18 month-old at home. The patient expressed concerns that her present illness could be Lyme disease. She had no recollection of a specific tick bite or rash, but did note she was often in close contact with tick-infested animals. Physical examination revealed painful range of motion of the left wrist and bilateral knees but no joint swelling, lymphadenopathy, rash or fever. Vital signs were normal. Initial CBC was normal except for low total WBC at 3.4 and a mild thrombocytopenia of 124,000. A Lyme titer was drawn. The patient was released from the ED with NSAIDs and instructed to follow up with her primary care physician to start doxycycline treatment pending the Lyme test results.

In clinic one week later, the patient presented with increased pain in bilateral hands, wrists, feet, ankles and knees. She had a new symptom of pruritus in the soles of her feet. The Lyme titer from the previous week returned negative. To expand the differential, 
further history was obtained. It was determined that her children had mild flu-like illnesses during the recent summer months, which brought to mind viral infections as causes of polyarthralgia. The sole positive finding on physical examination was her painlimited range of motion in her hands. Based on the key history element of recent sick contacts with young children and the clinical presentation, a parvovirus B19 serum antibody test and CBC was ordered. Her parvovirus antibodies confirmed her diagnosis of parvovirus B19 infection. See Table 1 for lab results.

\begin{tabular}{|c|c|c|c|}
\hline \multicolumn{5}{|c|}{ Table 1: Laboratory Test Results } \\
\hline Test & Initial Value & Value After One Week & Reference Value \\
\hline WBC & $\mathbf{3 . 4}$ & 4.7 & $4.0-11.0 \mathrm{~K} / \mu \mathrm{L}$ \\
\hline HGB & 11.9 & 12.3 & $12.0-16.0 \mathrm{~g} / \mathrm{dL}$ \\
\hline HCT & 36.2 & 37.2 & $36.0-48.0 \%$ \\
\hline Platelet Count & $\mathbf{1 2 4}$ & 170 & $150-400 \mathrm{~K} / \mu \mathrm{L}$ \\
\hline ESR & 5 & 4 & $0-20 \mathrm{~mm} / \mathrm{hr}$ \\
\hline RBC & 4.16 & 4.31 & $4.0-5.6 \mathrm{M} / \mu \mathrm{L}$ \\
\hline MCV & 87 & 86.3 & $82.0-100.0 \mathrm{fL}$ \\
\hline MCHC & 33 & 33 & $32.0-36.0 \mathrm{~g} / \mathrm{dL}$ \\
\hline MCH & 28.7 & 28.5 & $28.3-34.3 \mathrm{pg}$ \\
\hline RDW & 13.5 & 14 & $11.0-16.0 \%$ \\
\hline MPV & 9.9 & 10.5 & $7.4-10.45 \mathrm{fL}$ \\
\hline PMN \# & 2.4 & 3 & $1.50-6.50 \mathrm{~K} / \mathrm{uL}$ \\
\hline PMN \% & 71 & 64.1 & $43.0-76.0 \%$ \\
\hline Lymphocyte \# & 0.6 & 1.2 & $0.70-3.20 \mathrm{~K} / \mathrm{uL}$ \\
\hline Lymphocyte \% & 18.1 & 26.3 & $15.0-43.0 \%$ \\
\hline Monocyte \# & 0.2 & 0.3 & $0.20-0.90 \mathrm{~K} / \mathrm{uL}$ \\
\hline Monocyte \% & 5.7 & 7 & $4.8-12.0 \%$ \\
\hline Eosinophil \# & 0.1 & 0.1 & $0.00-0.500 \mathrm{~K} / \mathrm{uL}$ \\
\hline Eosinophil \% & 4.1 & 1.4 & $0.0-5.2 \%$ \\
\hline Basophil \# & 0 & 0.1 & $0.00-0.100 \mathrm{~K} / \mathrm{uL}$ \\
\hline Basophil \% & 1.1 & 1.2 & $0.0-2.5 \%$ \\
\hline Lyme Serology & Negative & - & Negative \\
\hline Parvovirus B19 IgM & - & $\mathbf{1 2 . 3}$ & $<0.9$ \\
\hline Parvovirus B19 IgG & - & $\mathbf{5 . 2}$ & $<0.9$ \\
\hline & & & \\
\hline
\end{tabular}

\section{Discussion}

The CDC estimates an annual incidence of clinician-diagnosed Lyme disease in the United States at 106.6 cases per 100,000 persons. ${ }^{i}$ With 136 confirmed cases of Lyme in West Virginia during 2014, fully 112 of these cases occurred in the eight eastern panhandle counties. This accounts for the inclination of eastern panhandle physicians to include Lyme disease early in differential diagnoses. Indeed, because the smaller nymph form is easily overlooked, only exposure to a potential tick habitat (rather than a documented tick bite) is required to meet the CDC definition of "exposure" in an endemic area like the eastern panhandle. ${ }^{\text {ii }}$ With this high incidence and low-threshold 
criteria in mind, it is reasonable to see how Lyme borreliosis would divert many practitioners in endemic areas from pursuing alternative diagnoses for a patient presenting with acute polyarthralgia. There is also a tendency to repeat the Lyme screen even in the face of a negative result in order to catch the common false-negative. It must be kept in mind, however, that though the Lyme screen sensitivity can be as low as $60 \%$ for early infections, the screening test is $97-100 \%$ accurate by the time polyarthralgia symptoms occur. ${ }^{\text {iii }}$ By not accepting the initial result and not eliminating Lyme from the differential, we incur unnecessary costs to the patient and run the risk of missing the true diagnosis. We additionally risk exposing the patient to unnecessary antibiotics by treating based on clinical suspicion alone. It is therefore proposed that, in a Lyme-endemic region when polyarthralgia is the complaint but the Lyme screen returns negative, the differential diagnosis be expanded to include mimicking conditions such as a parvovirus B19 infection.

Unlike Lyme borreliosis, it is difficult to estimate the true incidence of parvovirus B19 in our region due to the occult nature of many infections. As many as $20 \%$ of infected individuals are considered asymptomatic and many adults only ever develop a selflimited, mild, flu-like illness. ${ }^{\text {iv }}$ However, in a clinical case similar to this one with a female patient of reproductive age, there must be a low threshold for parvovirus B19 testing. Parvovirus infections in pregnant women can carry a risk of significant congenital defects in the unborn infant including fetal anemia and fetal hydrops. ${ }^{\text {iv }}$ Sample serologic data reports a seroconversion rate among pregnant women at $1.5 \%$ at baseline and as high as $13 \%$ during outbreak periods which are estimated to occur every 3-6 years. ${ }^{\text {iv }}$ In a Lyme-endemic region especially, it would be easy to mistake the clearance of the prodrome and polyarthralgia symptoms after misdiagnosed-Lyme treatment as confirmation of successful diagnosis and management rather than the natural course of a parvovirus B19 infection. This could subsequently lead to a missed diagnosis of a prenatal exposure to a high-risk intrapartum infection. It is therefore suggested that all similarly presenting patients with an acute polyarthropathy suspicious for parvovirus B19 be screened for pregnancy at some point during the course of their workup.

\section{Conclusion}

Consideration of this case presents an argument for expanding the differential diagnosis in patients with acute polyarthralgia and investing more store into the clinical presentation and history to avoid unnecessary testing, treatment and, ultimately, expense. Though the patient identifies herself as a high-risk individual for contracting Lyme disease, her presentation and lab serology were not consistent with this diagnosis and it was necessary to expand the differential diagnosis to include alternatives such as parvovirus B19. It is additionally important to remember parvovirus B19 as a possible etiology of painful arthralgias especially in the pregnant population. 


\section{References}

i Nelson CA, Saha S, Kugeler KJ, Delorey MJ, Shankar MB, Hinckley AP, et al. Incidence of cliniciandiagnosed Lyme disease, United States, 2005-2010. Emerg Infect Dis. 2015 Aug

ii Lyme disease (Borrelia burgdorferi) 2011 Case Definition [Internet]. National Notifiable Disease Surveillance System. Centers for Disease Control and Prevention; Available from: https://wwwn.cdc.gov/nndss/conditions/lyme-disease/case-definition/1996/

iii Johnson BJB. Laboratory Diagnostic Testing for Borrelia burgdorferi Infection. In: Lyme Disease: An Evidence-based Approach. CAB International; 2011. p. 73-88.

${ }^{i v}$ Lamont R, Sobel J, Vaisbuch E, Kusanovic J, Mazaki-Tovi S, Kim S, et al. Parvovirus B19 infection in human pregnancy. BJOG: An International Journal of Obstetrics \& Gynaecology. 2010;118(2):175-86. 